\title{
The Bivalency Effect in Task Switching: Event-Related Potentials
}

\author{
John G. Grundy,' Miriam F.F. Benarroch,' Todd S. Woodward, ${ }^{2,3}$ \\ Paul D. Metzak, ${ }^{2,3}$ Jennifer C. Whitman, ${ }^{2,3}$ and Judith M. Shedden ${ }^{1 *}$ \\ ${ }^{1}$ Psychology, Neuroscience and Behaviour, McMaster University, Ontario, Canada \\ ${ }^{2}$ Department of Psychiatry, University of British Columbia, Vancouver, British Columbia, Canada \\ ${ }^{3}$ BC Mental Health and Addiction Research Institute, Vancouver, British Columbia, Canada
}

\begin{abstract}
During task switching, if we occasionally encounter stimuli that cue more than one task (i.e., bivalent stimuli), response slowing is observed on all univalent trials within that block, even when no features overlap with the bivalent stimuli. This observation is known as the bivalency effect. Previous fMRI work (Woodward et al., 2008) clearly suggests a role for the dorsal anterior cingulate cortex (dACC) in the bivalency effect, but the time course remains uncertain. Here, we present the first hightemporal resolution account for the bivalency effect using stimulus-locked event-related potentials. Participants alternated among three simple tasks in six experimental blocks, with bivalent stimuli appearing occasionally in bivalent blocks (blocks 2, 4, and 6). The increased reaction times for univalent stimuli in bivalent blocks demonstrate that these stimuli are being processed differently from univalent stimuli in purely univalent blocks. Frontal electrode sites captured significant amplitude differences associated with the bivalency effect within time windows 100-120 ms, 375-450 ms, and 500-550 ms, which may reflect additional extraction of visual features present in bivalent stimuli (100-120 ms) and suppression of processing carried over from irrelevant cues (375-450 ms and 500-550 ms). Our results support the fMRI findings and provide additional evidence for involvement of the dACC. Furthermore, the bivalency effect dissipated with extended practice both behaviorally and electrophysiologically. These findings are discussed in relation to the differential processing involved in a controlled response style. Hum Brain Mapp 34:999-1012, 2013. @ 2011 Wiley Periodicals, Inc.
\end{abstract}

Key words: event-related potentials; bivalency effect; task-switching; ERP; EEG

Contract grant sponsor: Natural Sciences and Engineering Research Council of Canada (NSERC) (JMS).

*Correspondence to: Dr. Judith M. Shedden, Department of Psychology, Neuroscience and Behaviour, McMaster University, 1280 Main Street West, Hamilton, ON L8S 4K1, Canada.

E-mail: shedden@mcmaster.ca

Received for publication 10 January 2011; Accepted 15 September 2011

DOI: $10.1002 / \mathrm{hbm} .21488$

Published online 8 December 2011 in Wiley Online Library (wileyonlinelibrary.com).

\section{INTRODUCTION}

The bivalency effect refers to a blockwise slowing of responses that occurs when occasional task stimuli contain cues from two ongoing tasks (bivalent stimuli). The appearance of even a few bivalent stimuli causes response slowing on all other trials within the block. Consider for instance, an experiment involving three tasks: a color task which requires classification of shapes as red or blue, a case task which requires classification of letters as uppercase or lowercase, and a parity task which requires classification of digits as odd or even. Now consider encountering a red letter. The colored letter is a bivalent stimulus because it contains features from both the letter 
task and the color task; the letter cues the participant to perform the case judgment task while its colored appearance cues the participant to perform the color-judgment task. Participants must ignore the color-judgment cue to make the correct case judgment response, and performance on the bivalent trials suffers. The bivalency effect refers to the observation that, during practiced performance of different tasks in alternation, the occasional presence of a bivalent trial affects performance on univalent trials within the same block, even when the features of the univalent stimuli do not overlap with the bivalent features (Woodward et al., 2003). A few bivalent stimuli within a block may be enough to elicit a blockwise response strategy requiring adjustment in control, and this adjustment in control appears to involve the anterior cingulate cortex (ACC; Woodward et al., 2008).

Activation of the ACC has consistently been reported in studies that compare task switching versus task repetition (e.g., Slagter et al., 2006; Yeung et al., 2006), as well as studies that use bivalent stimuli to stimulate processing conflict (Kerns et al., 2004; Liu et al., 2004; Milham et al., 2001; van Veen et al., 2001). Experiments that examine the bivalency effect, including the experiment described in this article, alternate between different tasks on each trial, so there is no contrast between switching and repeat trials. However, the involvement of the ACC in the bivalency effect can still be evaluated. The frontal ACC is more strongly activated for univalent trials within blocks that include a few bivalent stimuli compared to univalent trials in purely univalent blocks (Woodward et al., 2008). This suggests that the frontal ACC is recruited when adjustment in control is triggered by the presence of a few bivalent stimuli. The authors characterized this as a change in response style that requires breaking the inertia that has built up during the presentation of purely univalent stimuli in the previous block (Woodward et al., 2008).

The fMRI results clearly point to a role for the ACC in the bivalency effect; however, due to the temporal limitations of fMRI, the time course of ACC involvement immediately after stimulus onset remains uncertain. This time course can be illuminated with event-related potentials (ERPs), which provide millisecond temporal resolution. We do not know whether the fMRI activity difference between univalent stimuli in bivalent blocks (hereafter referred to as uni-biv stimuli) and univalent stimuli in purely univalent blocks (hereafter referred to as uni-uni stimuli) is a result of ACC response differences at early or late stages within the time course following the stimulus. Higher resolution temporal measurements from ERP will help to answer this question and clarify the role of the ACC in the bivalency effect.

As far as we know, the current article is the first ERP work examining the bivalency effect, per se. However, even though the three tasks alternate on every trial in bivalency effect studies (i.e., there are no task repeat trials), it is expected that important processing might be reflected in similar ERP components reported in other task switching studies that compare switching trials to repeat trials. The task switch versus repeat contrast within an ERP study typically reveals a negative-going waveform for switch trials relative to repeat trials over parietal electrode $\mathrm{Pz}$ that begins between 300 and $400 \mathrm{~ms}$ after stimulus onset (e.g., Karayanidis et al., 2003; Hsieh and Liu, 2008). Studies comparing switch to repeat trials have also found ERP differences in frontal areas that may reflect ACC involvement in task switching (Slagter et al., 2006; Wylie et al., 2003; Yeung et al., 2006). For example, Wylie, Javitt, and Foxe (2003) found a more negative deflection for switch trials relative to repeat trials at frontal electrode sites (F3 and F4) 470 ms after stimulus onset. Other studies have found task switch related negativity at frontal electrode sites between 350 and $600 \mathrm{~ms}$ (Lorist, et al., 2000), 300-600 ms (Hsieh and Liu, 2009), and 400-700 ms (Hsieh and Chen, 2006).

When comparing task switching to task repetition, there is some reconfiguration required to adjust stimulus evaluation and responses to the new task, and this is often associated with ACC involvement (e.g., Wylie et al., 2009). In the bivalency effect, all the trials are switching trials and participants are well trained on the alternating task sequence so that the additional control associated with the ACC may be lessened. When a bivalent stimulus is suddenly encountered, even though the tasks and the task sequence have not changed, the ACC may reengage on all trials to sort out the general conflict generated by the occasional irrelevant stimulus feature. If the ACC is influencing these same task-switching processes to produce the bivalency effect, then we might expect to distinguish univalent stimuli in bivalent blocks relative to univalent stimuli in univalent blocks over frontal electrodes within the same time windows reported by the task switching ERP studies mentioned earlier. Although the slowing of responses in bivalent blocks does not exactly parallel the difference between task switch and repeat trials, the ACC may influence the same critical task switching processes to bring about the bivalency effect. We would also predict that with enough practice with the bivalent stimuli, the ACC involvement would once again be lessened; therefore, the frontal activity that we predict will distinguish between univalent stimuli in univalent versus bivalent blocks should lessen with extended practice.

Task-switching studies that deal with stimulus conflict and/or response conflict may also help predict the time course of ERP differences associated with the bivalency effect. For example, modulation of the N2 ERP component is associated with task stimuli that contain some form of conflict (Yang and Wang, 2002; Kong et al. 2000; Wang et al., 2002). The N2 typically occurs between 200 and $350 \mathrm{~ms}$ after stimulus onset, is recorded at frontal electrode sites and has been indexed to the ACC (van Veen and Carter, 2002), an area that neuroimaging studies link to the bivalency effect. ERP studies that directly compare univalent trials to bivalent trials within a task-switching paradigm have reported frontopolar ERP differences 
between 300 and 600 ms (Poulsen et al., 2005; Hsieh and Liu, 2008). The contrast in the bivalency effect is between univalent stimuli that occur in univalent blocks versus bivalent blocks and does not compare univalent to bivalent trial responses directly (less than $7 \%$ of the trials in the bivalent blocks are bivalent trials); however, the ACC activity associated with the need to adjust control in the bivalent blocks may be similar.

We recorded stimulus-locked ERPs while participants alternated between a color judgment of a shape (red vs. blue), a parity judgment of a digit (odd vs. even), and a case judgment of a letter (uppercase vs. lowercase). The trials were grouped into univalent blocks in which no bivalent stimuli appeared (i.e., purely univalent blocks), and bivalent blocks in which bivalent stimuli appeared on $20 \%$ of the case judgment trials (uppercase or lowercase letters in red or blue). Our focus of interest is on ERP activity that corresponds with the ACC activity reported in the fMRI experiments (Woodward et al., 2008), and the change in the ACC activity with extended practice. Past studies on the bivalency effect have analyzed three alternating experimental blocks (Woodward et al., 2003, 2006, 2008). These blocks consisted of a bivalent block flanked by two univalent blocks; the experimental blocks were preceded by univalent practice blocks. This study added three additional alternating experimental blocks to assess the effects of extensive practice on the bivalency effect. We expected to see a decrease in the bivalency effect over time as participants learned to process the bivalent stimuli more efficiently, and a corresponding decrease in ACC-related ERP responses. Previous work has shown that the bivalency effect is reduced when participants know to expect bivalent stimuli (Metzak et al., submitted). Furthermore, Meier et al. (2009) demonstrated a decrease in the bivalency effect on subsequent trials following bivalent stimuli. This suggests that extending the number of experimental blocks may also result in a reduction of the bivalency effect.

\section{METHODS}

\section{Participants}

Twenty-five undergraduate volunteers (eight males) from McMaster University's Introductory Psychology and Cognition subject pool participated for course credit. All participants had normal or corrected-to-normal vision. All procedures complied with the Canadian tri-council policy on ethics and were approved by the McMaster Ethics Research Board.

\section{Materials and Apparatus}

Stimuli were presented on a black background on a $17^{\prime \prime}$ CRT monitor (resolution $1024 \times 768$; refresh rate $85 \mathrm{~Hz}$ ). The experiment was controlled by Presentation ${ }^{\circledR}$ Software (Neuro Behavioral Systems; version 11). The stimulus set

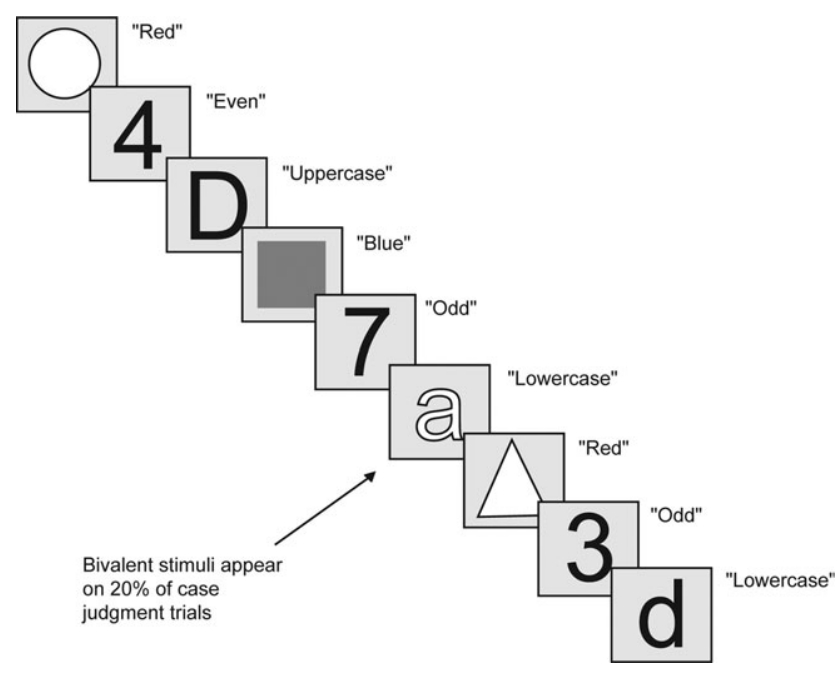

Figure I.

Illustration of the trial sequence and type of stimuli used during the experiment. The words appearing beside the stimuli represent correct responses. During bivalent blocks (blocks 2, 4, and 6), bivalent stimuli appear on $20 \%$ of all case-judgment trials. Bivalent stimuli do not appear at all during the univalent blocks (blocks I, 3, and 5). In the gray-scale diagram, we use white to represent the red stimuli and gray to represent the blue stimuli.

was adapted from Woodward et al. (2008). For the color decision task, the stimuli were one of four shapes (circle, triangle, square, or pentagon), displayed in either red or blue. The parity decision task consisted of numerals 1-8, displayed in white (60-point Times New Roman font). The case decision task consisted of letters $a, b, d$, and e, displayed in uppercase or lowercase (white, 60-point Times New Roman). For the bivalent stimuli, the case letters were displayed in either red or blue (e.g., lowercase "a" displayed in blue). The color of the bivalent stimuli was randomly varied between red and blue. This resulted in some bivalent stimuli in which the case judgment response was congruent with the color response and other bivalent stimuli in which the case judgment response was incongruent with the color response. Participants were seated in a dimly lit room at a distance of $90 \mathrm{~cm}$ from the monitor, with the height of each stimulus subtending a visual angle of $1.26^{\circ}$. A chinrest was used to ensure that each participant was viewing the screen from the same distance, and to minimize movement artefacts during ERP recordings. See Fig. 1 for an example of the stimuli and sequence of trials.

\section{Procedure}

Before participation, written informed consent was obtained from all participants. Each experimental session was $\sim 2 \mathrm{~h}$ in duration, including electrode application. Participants were informed that they would be completing 
three tasks in alternation and that they should indicate with a key press whether the shape was red or blue (color-judgment task), whether the number was odd or even (parity-judgment task), and whether the letter was uppercase or lowercase (case-judgment task). Participants were instructed to make a left key press (the " $<$ " key using index finger of right hand) for blue shapes, odd digits, and lowercase letters, and to make a right key press (the " $>$ " key using middle finger of right hand) for red shapes, even digits, and uppercase letters. Left and right key mappings were counter-balanced across participants. The sequence of trials always proceeded from color to parity to case. Participants were not informed that bivalent stimuli would appear.

Past experiments have shown that accuracy on the three tasks is typically quite high (Woodward et al., 2003, 2006, 2008), and so we imposed a time limit of $1500 \mathrm{~ms}$ to encourage the participants to respond quickly as well as accurately. This time restriction is also in line with the procedure used by Woodward et al. (2008) in their fMRI study of the bivalency effect. On each trial, a stimulus (shape, digit, or letter) was presented in the center of the computer screen (Fig. 1) and remained visible until the participant responded or for $1,500 \mathrm{~ms}$, at which point the message "Too slow!" appeared on the screen. The intertrial interval was randomized between 400 and $900 \mathrm{~ms}$ throughout the experiment. The varying intertrial interval was used to reduce distortion of ERP components due to averaging of event epochs that overlap in time (Woldorff, 1993). To reduce blinking and general movement that might interfere with our ability to observe task-relevant ERPs, a message appeared after every 12 trials indicating that participants could take a "blink break." To maintain motivation, accuracy over the previous 12 trials was provided during the blink break. Breaks were also provided between blocks. Participants resumed the experiment by pressing one of the response keys to start the next trial.

There were eight blocks, each consisting of 168 trials (two practice blocks followed by six experimental blocks). There were two types of blocks. The two practice blocks and experimental blocks 1, 3, and 5 were univalent blocks consisting of purely univalent stimuli. The 168 univalent stimuli in each of the univalent blocks, consisted of 56 color-judgment stimuli, 56 parity-judgment stimuli, and 56 case-judgment stimuli (presented in predictable order of color, parity, and case). Experimental blocks 2, 4, and 6 were bivalent blocks, which were similar to the univalent blocks except that on a random 16 of the 56 case-judgment trials, the stimulus letters were presented in red or blue (bivalent trials). The color of the letters was irrelevant to the case-judgment response.

Analyses examining the bivalency effect were restricted to univalent trials. The 16 bivalent trials in the bivalent blocks were not included in these analyses. We will use "uni-uni" to refer to univalent trials in univalent blocks and "uni-biv" to refer to univalent trials in bivalent blocks. Two types of reaction time (RT) analyses were per- formed to examine task type (color, parity, case) and block type (univalent vs. bivalent). One analysis compared uniuni trials from experimental blocks 1 and 3 to uni-biv trials from experimental block 2 across the three tasks, consistent with fMRI analyses (Woodward et al., 2008), under the hypothesis that uni-biv trials would show slower response times than uni-uni trials. The other RT analyses compared uni-uni trials to uni-biv trials across all six experimental blocks. We hypothesized that the magnitude of the bivalency effect would be reduced with practice; therefore, we compared uni-uni and uni-biv differences across block pairs. Block 1 mean RTs were subtracted from block 2 means (blocks 2-1), block 3 means were subtracted from block 4 means (blocks $4-3$ ), and block 5 means were subtracted from block 6 means (blocks 6-5). If the bivalency effect is reduced with practice, then the RT mean differences for blocks 2-1 should be larger than the mean differences for blocks 4-3 and blocks 6-5. The second analysis asked whether the bivalency effect dissipated more slowly for the color task compared to the case and parity task. We used a polynomial contrast with five levels to measure performance across the six-block sequence to look for an interaction between task and the bivalency effect over time. Following a significant interaction, we then compared the tasks more directly with pairwise comparisons.

\section{Electrophysiological Recordings and Analyses}

The ActiveTwo Biosemi electrophysiology system (www.biosemi.com) was used to record continuous electroencephalographic (EEG) activity from $128 \mathrm{Ag} / \mathrm{AgCl}$ scalp electrodes plus four additional electrodes placed at the outer canthi and just below each eye for recording of horizontal and vertical eye movements. Two additional electrodes, a common mode sense active electrode and a driven right leg passive electrode were also used. These electrodes replace the "ground" electrodes used in conventional systems (www.biosemi.com/faq/cms\&drl.htm). The continuous signal was acquired with an open passband from DC to $150 \mathrm{~Hz}$ and digitized at $512 \mathrm{~Hz}$. The signal was bandpass filtered offline at 0.3 to $30 \mathrm{~Hz}$ and re-referenced to a common average reference. Offline signal processing and averaging were done using EEProbe (www.antnero.com). Voltage maps were created using Brain Electrical Source Analysis (BESA; version 5.1.8; www.besa.de) by MEGIS Software GmbH. Eye blinks and movement artifacts were automatically identified and manually verified. EEProbe signal processing software was used to apply a correction procedure; eye movement prototypes were estimated for each individual and movement artifacts were subtracted across the electrode array based on calculated vertical electro-oculogram (VEOG) propagation factors via a regression algorithm. Each corrected waveform was verified manually; epochs containing eye-blinks or movements that could not be adequately corrected were rejected from the analyses. 
EEG analysis allows for a vast number of possible comparisons between locations, times, and conditions. To reduce the search space, we used a multivariate statistical tool, partial least squares (PLS; Lobaugh et al., 2001; McIntosh et al., 1996), which does not require any a priori bias with respect to time course or location of effects. PLS is similar to a principle components analysis (PCA) in that it uses singular value decomposition to extract information from the dataset, but different in that it constrains the analysis to variance that can be explained by experimental conditions. Singular value decomposition yields a set of latent variables (LVs; similar to eigenvalues in PCA) that represent particular contrasts, which account for a percentage of the cross-block covariance explained by the experimental conditions. Each singular value explains how much of the covariance was explained by a particular LV. One thousand permutations were computed and provided an estimate of obtaining a singular value by chance (similar to a $P$ value). The electrode saliences represent the relation between the experimental design contrasts (as represented by the LV) and the spatiotemporal pattern of ERP amplitude changes. Two hundred bootstrap resamplings were performed to assess the reliability of electrode saliences at each time point by providing a standard error for each salience. The bootstrap procedure uses random sampling with replacement so that even though each sample will have the same number of elements as the original data, slightly different samples will be produced and reliability of the saliences can be measured. As the ratio of the salience to the standard error is approximately equal to a $z$ score, data points where the ratio was more than $1.7(P<$ $0.05)$ were considered reliable. For a nice example of how PLS can be applied to EEG data, see Düzel et al. (2003).

The PLS analyses allowed us to narrow the time windows and locations of experimental effects in order to perform conventional statistics. Our primary area of interest remains the frontal areas as we aim to address the role of the ACC in the bivalency effect. As such, we will be primarily restricting our analysis to electrophysiological differences observed within frontal electrodes. Repeated measures analyses of variance (ANOVAs) and paired samples $t$-tests were performed on specific time points and locations of interest.

Dipole source analysis was performed on the ERP difference scores between uni-uni trials and uni-biv trials to spatially localize where the bivalency effect was being captured for all time windows of interest. Using BESA software (version 5.1.8), a four-shell spherical head model (head, scalp, bone, and cerebrospinal fluid) was used as an approximation of dipole fitting. The scalp thickness was set to $6 \mathrm{~mm}$, and the bone thickness was set to $7 \mathrm{~mm}$.

PCA was used to determine the number of dipoles to be fitted for each model (e.g., each time window). Dipoles explaining less than $2 \%$ of the variance were not considered. Locations and orientations of the dipoles were calculated to account for the maximum amount of variance that could be explained within a particular time window. The residual variance (RV) expresses the percentage of the ERP power not explained by the fitted dipoles.

\section{RESULTS}

\section{Behavioural Results: Accuracy}

Although RT data is our focus behaviorally, we will first present accuracy data to rule out speed-accuracy tradeoffs.

Accuracy was tested in two different ways. The first set of analyses was performed on the first three experimental blocks, consistent with the fMRI analyses in Woodward et al. (2008). A $2 \times 3$ omnibus repeated measures ANOVA crossed block (average of uni-uni trials in blocks 1 and 3 vs. uni-biv trials in block 2) and task (color, parity, and case) to examine accuracy within the first three blocks. There was a significant main effect of block, $F(1,24)=$ 7.50, $P=0.011, \eta^{2}=0.238$, and a significant main effect of task, $F(2,48)=12.69, P<0.0001, \eta^{2}=0.346$, but no interaction, $F(2,48)=0.795, P=0.458, \eta^{2}=0.032$. Pairwise tests indicate that a higher proportion of errors were made during the first bivalent block than in the average of blocks 1 and $3, t(24)=3.376, P=0.002$, and that the case task had fewer errors than the parity and color tasks, $t(24)$ $=4.15, P<0.001$, and $t(24)=4.25, P<0.001$, respectively; parity and color did not differ from each other $t(24)=$ $0.114, P=0.910$.

\section{Behavioural Results: Accuracy and Extended Practice}

Extended practice effects on the proportion of errors were analyzed by examining the difference scores of blocks 1 and 2, blocks 3 and 4, and blocks 5 and 6, using task as an additional within-subject factor. A $3 \times 3$ (task X block difference scores) repeated-measures ANOVA was performed and revealed a significant main effect of block differences, $F(2,48)=6.38, P=0.003, \eta^{2}=0.210$. There was no main effect of task, $F(2,48)=1.91, P=0.159, \eta^{2}=$ 0.074 , and no interaction, $F(4,96)=0.896, P=0.470, \eta^{2}=$ 0.036 . The significant main effect of block differences can be explained by pairwise comparisons indicating that block 2-1 and block 4-3 showed larger block differences for proportion of errors than block $6-5, t(24)=2.92, P=$ $0.008, t(24)=2.67, P=0.013$, respectively, but block $2-1$ and block 4-3 did not differ from each other, $t(24)=$ $-1.12, P=0.272$. This is consistent with a dissipating error rate given extended practice.

\section{Behavioural Results: The Bivalency Effect (RTs to Uni-Biv Trials vs. Uni-Uni Trials)}

All mean RTs for task type and block are illustrated in Fig. 2; all error trials were excluded from RT analyses. For repeated measures analyses of factors involving more than 


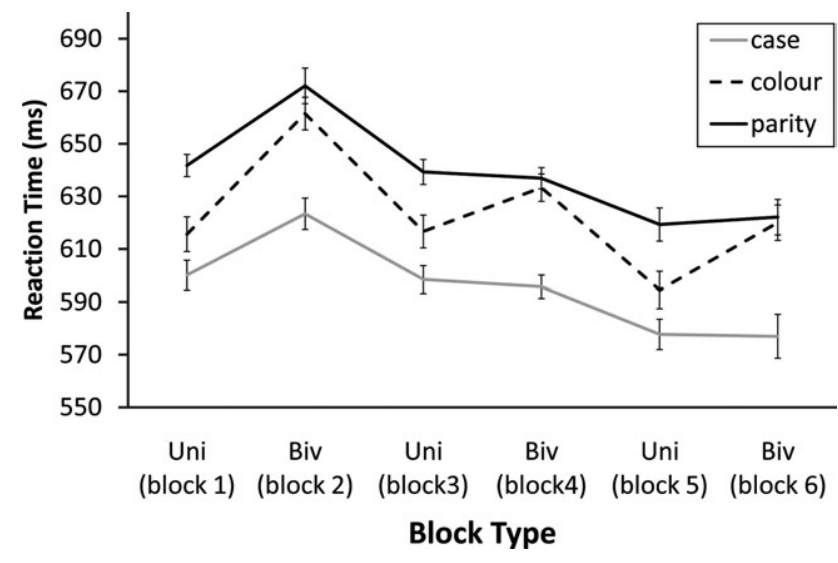

Figure 2.

Mean reaction times for univalent trials in all six experimental blocks for the three tasks: case, color, and parity. Error bars represent standard errors.

two levels, the Greenhouse-Geisser correction was applied when necessary, in which case epsilon and the adjusted $P$ values are reported along with the original degrees of freedom.

Like the accuracy data, the bivalency effect on RTs was tested in two different ways. The first set of analyses was performed on the first three experimental blocks, consistent with the fMRI analyses in Woodward et al. (2008). A 2 $\times 3$ omnibus repeated measures ANOVA crossed block (average of uni-uni trials in blocks 1 and 3 vs. uni-biv trials in block 2) and task (color, parity, and case) to examine the bivalency effect within the first three blocks. The analysis revealed a significant main effect of task, $F(2,48)=$ 6.54, $P=0.006, \eta^{2}=0.214$, a significant main effect of block, $F(1,24)=29.21, P<0.0001, \eta^{2}=0.549$, and a significant interaction, $F(2,48)=6.68, P=0.003, \eta^{2}=0.218$. The significant block effect shows that uni-biv trials were slower than uni-uni trials, as predicted. Pairwise comparisons for the tasks revealed that participants were faster at responding to the case task compared to both the color task, $t(24)=2.619, P=0.015$, and the parity task, $t(24)=$ $5.568, P<0.001$, but that there were no response time differences between the color and parity tasks, $t(24)=1.149$, $P=0.262$. Follow-up analyses comparing uni-uni trials in blocks 1 and 3 to uni-biv trials in block 2 for each task confirmed that the bivalency effect was present in the colour task, $F(1,24)=47.56, P<0.0001, \eta^{2}=0.665$, the parity task, $F(1,24)=22.2, P<0.0001, \eta^{2}=0.488$ and the case task, $F(1,24)=10.37, P=0.004, \eta^{2}=0.302$. To clarify the interaction, pairwise comparisons on the difference scores (uni-biv trials in block 2 minus uni-uni trials in blocks 1 and 3) for each task revealed that the magnitude of the bivalency effect was greatest for the color task compared to the case task, $t(24)=4.32, P<0.001$, and the parity task, $t(24)=2.91, P=0.008$ but did not differ between the case and parity tasks, $t(24)=1.39, P=0.193$.

\section{Behaviour: Extended Practice and the Bivalency Effect (RTs to Uni-Biv Trials vs. Uni-Uni Trials)}

The second set of analyses included all six experimental blocks to examine questions about practice effects. An analysis of RT differences between pairs of univalent and bivalent blocks (univalent trials only) was performed to assess whether the magnitude of the bivalency effect changes with practice. Extensive practice effects were analyzed by examining the difference scores of blocks 1 and 2, blocks 3 and 4, and blocks 5 and 6. Task was included as a factor to assess possible differences of practice on the bivalency effect across task. The difference scores (blocks 2-1, 4-3, and 6-5) were analyzed by means of a $3 \times 3$ repeated measures ANOVA (block differences $X$ task). Significant main effects of task type and block differences were revealed, $F(2,48)=19.87, P<0.0001, \eta^{2}=0.453, F(2$, $48)=6.30, P=0.004, \eta^{2}=0.208$, respectively. Task did not interact with block differences, $F(4,96)=0.21, P=0.932$, $\eta^{2}=0.208$ indicating that the effect of practice on the bivalency effect did not differ across task. The main effect of task type can be explained by pairwise comparisons indicating that the difference scores were larger for the color task compared to the case task, $t(24)=5.27, P<$ 0.001 , and the parity task, $t(24)=5.69, P<0.001$ but did not differ between the case and the parity task, $t(24)=$ $1.01, P=0.324$. Pairwise comparisons for the block differences (bivalency effect) revealed that RT differences between blocks 2-1 were larger than the differences in blocks $4-3, t(24)=3.19, P=0.004$, and blocks $6-5, t(24)=$ $5.69, P<0.001$, but that the difference scores for blocks $4-3$ and 6-5 were not significantly different from each other, $t(24)=0.475, P=0.639$. These results are consistent with a dissipating bivalency effect across the experiment.

Although the interaction between block pair differences and task was not significant, it is interesting that there was a larger bivalency effect for the color task compared to case and parity tasks in the previous analyses. The irrelevant feature in the bivalent stimulus was color, leading to a question of whether there might be a trend for the dissipation of the bivalency effect to occur more slowly for the color task. One way to ask this question is to use a polynomial trend analysis with five levels (order 5 contrast) to look at the pattern of RTs sequentially across all six blocks. This model defines the hypothesis that the mean response time will change on each sequential block: slower responses on block 2 compared to block 1, faster responses on block 3 compared to block 2, and continuing in this pattern as blocks alternate between univalent and bivalent, producing five changes in direction. This analysis can be run as a $6 \times 3$ repeated measures ANOVA with block (1, $2,3,4,5$, and 6) crossed with task (color, parity, and case), and should produce a significant interaction if the RT trend across the six blocks differs between the tasks. The ANOVA analysis revealed a significant main effect of block, $F(5,120)=10.98, e=0.58, P<0.001, \eta^{2}=0.314$, a significant main effect of task, $F(2,48)=7.99, e=0.76, P$ 
$=0.003, \eta^{2}=0.250$, and a significant interaction, $F(10,240)$ $=2.87, e=0.62, P=0.01, \eta^{2}=0.107$. The within-subject contrasts produced a significant order 5 contrast for block, $F(1,24)=18.7, P<0.001, \eta^{2}=0.437$, and a significant order 5 contrast for the interaction between block and task, $F(1,24)=15.7, P=0.001, \eta^{2}=0.395$, suggesting that the trend differed between the tasks. To test the hypothesis that the color task differed from the other two tasks in terms of the dissipation of the bivalency effect with practice, pairwise comparisons between the color task and the average of the parity and case tasks were conducted on the block pair differences. The RT difference between uniuni and uni-biv trials was larger for the color task compared to the parity and case tasks for the block 2-1 difference (46.1 vs. 30.0, respectively; $t(24)=2.86, P=0.009$ ), for the block $4-3$ difference (17.0 vs. 0.9 , respectively; $t(24)$ $=2.21, P=0.037)$, and for the block 6-5 difference ( 23.7 vs. 3.3 , respectively; $t(24)=4.00, P=0.001)$, supporting the hypothesis that the bivalency effect dissipates more slowly for the colour task compared to the parity and case tasks.

\section{Electrophysiological Results}

The average RT across all blocks was $\sim 650 \mathrm{~ms}$; therefore, a -200 - to $600-\mathrm{ms}$ time window was used to capture stimulus-locked ERPs for all analyses. We first examine the bivalency effect (uni-uni trials compared to uni-biv trials) via whole-brain PLS analysis followed by a classic statistical analysis of ERP amplitudes across the first three experimental blocks. We then discuss the effect of extended practice on the bivalency effect across all six experimental blocks. Only significant LVs identified by PLS are discussed.

\section{Electrophysiology: Bivalency Effect (Uni-Uni vs. Uni-Biv Trials)}

The PLS analysis was conducted to examine the bivalency effect. An examination of latent variable 1 (LV1) suggested that univalent trials in the first bivalent block (block 2) were processed differently from univalent trials in other blocks (see Fig. 3A) and accounted for 35\% of the variance, $P=0.006$. The bootstrap analysis of electrode salience, which provides confidence intervals for salience across time points and electrodes, revealed that this effect was most reliable in frontal electrodes (Fpz, AFz, AF4, and AF3; hereafter referred to as the frontal cluster; see Fig. 4) within time windows 115-135 ms, 300-340 ms, and 500$580 \mathrm{~ms}$. These results are consistent with fMRI results (Woodward et al., 2008) for which univalent trials in the first bivalent block (block 2) showed differential activation from that of univalent trials in the first two univalent blocks (blocks 1 and 3) in the frontal ACC. Even though this difference was not captured by central sites $(\mathrm{Cz}, \mathrm{C} 1$, $\mathrm{C} 2, \mathrm{FCz}, \mathrm{FC} 1$, and FC2; hereafter referred to as the central cluster; see Fig. 4) as might be expected from ERP studies that discuss ACC activity (e.g., Stemmer et al., 2003; Ullsperger and von Cramon, 2001; van Veen and Carter, 2002), the fMRI results (Woodward et al., 2008) showed a very frontal ACC BOLD response that is consistent with our frontal cluster (see Fig. 5 for topographies and Fig. 6 for source models). Examination of LV1 also suggested that ERP responses to univalent trials in the later bivalent blocks did not differ from responses in the univalent blocks, which supports the behavioral results showing that the bivalency effect dissipates over the course of the experiment. To examine these results further, classic componential statistical tests were performed on the bivalency effect at the frontal cluster, and for comparison at the central cluster. Components were selected based on visual inspection and correspondence with the PLS results.

The frontal electrode cluster captured the bivalency effect (mean of blocks 1 and 3 compared to block 2) within time windows $100-120 \mathrm{~ms}, 375-450 \mathrm{~ms}$, and $500-550 \mathrm{~ms}$ (see Fig. 3B). Paired-samples $t$-tests revealed that uni-uni trials showed more negative amplitudes than uni-biv trials at $100-120 \mathrm{~ms}(-2.39 \mu \mathrm{V}$ vs. $-2.12 \mu \mathrm{V}$, respectively; $t(1,24)$ $\left.=-2.76, P=0.011, \eta^{2}=0.241\right)$, and less negative amplitudes at $375-450 \mathrm{~ms}(-4.69 \mu \mathrm{V}$ vs. $-5.24 \mu \mathrm{V}$, respectively; $\left.t(1,24)=-2.639, P=0.014, \eta^{2}=0.225\right)$ and $500-550 \mathrm{~ms}$ $(-3.19 \mu \mathrm{V}$ vs. $-3.71 \mu \mathrm{V}$, respectively; $t(1,24)=3.518, P=$ $\left.0.002, \eta^{2}=0.340\right)$. No significant electrophysiological differences were found over the central cluster (see Fig. 3B). The voltage maps (Fig. 5) illustrate the frontal extent of the bivalency effect contrast, consistent with the frontal ACC activity shown by the fMRI results (Woodward et al., 2008).

To provide further confidence in our claim that these results are consistent with fMRI results, we performed source dipole analysis (BESA) on the amplitude differences between uni-biv and uni-uni trials. We used PCA to determine the number of dipoles needed for each time window; the software then automatically calculated the locations and orientations of each dipole. All coordinates are reported in Talairach-Tournoux coordinates.

For the 100-120-ms time window, two principle components were needed to account for $99.8 \%$ of the variance $(95.4 \%+4.4 \%$ separately) in the ERP difference scores. The first dipole (accounting for $95.4 \%$ of the variance) was located at $x, y, z=-27.5,-53.5,11.2$, orientation (ori): $-0.5,-0.4,-0.7$. The second dipole was located at $x, y, z$ $=44.3,-31,7.2$, ori: $0.4,-0.8,-0.4$. This model is the best fit for the data, and corresponds to a residual variance (RV) of $5.5 \%$ at the peak activity of these dipoles (100 ms; see Fig. 6A). We will focus on the first dipole which is located in the left temporal region. Our hypothesis is that this dipole is explaining variance due to both posterior and anterior activity, and it is possible that the 100-120 ms effect we have measured at the frontal cluster is reflecting simultaneous activity in more posterior sites. Examination of a cluster of electrodes (P5, P7, and PO7) over the left temporal parietal scalp area supports this hypothesis. 


\section{PLS Univalent trials only}

A

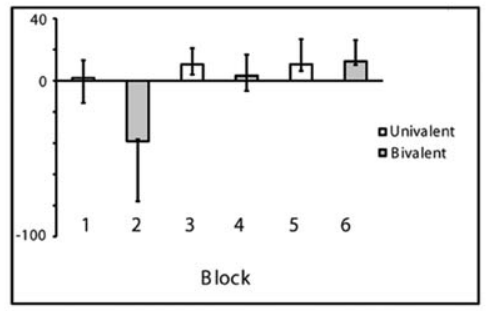

Frontal cluster

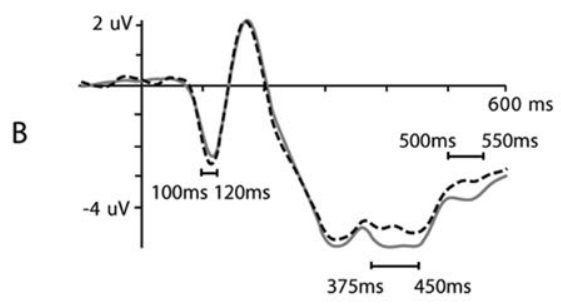

\section{Central cluster}

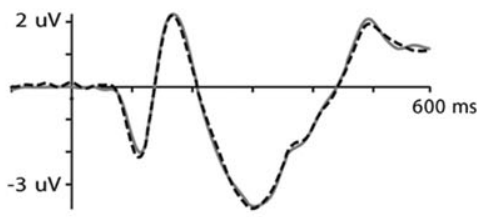

---- Univalent trials (block 2) __ Univalent trials (blocks 1 and 3 )

\section{Frontal clus ter}

Univalent trials only $100-120 \mathrm{~ms}$

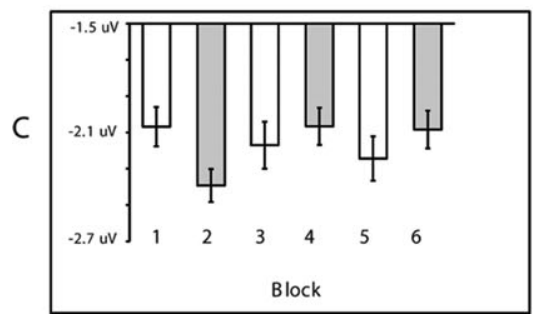

Univalent trials only $375-450 \mathrm{~ms}$

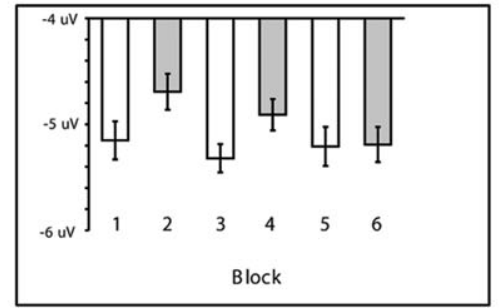

Univalent trials only $500-550 \mathrm{~ms}$

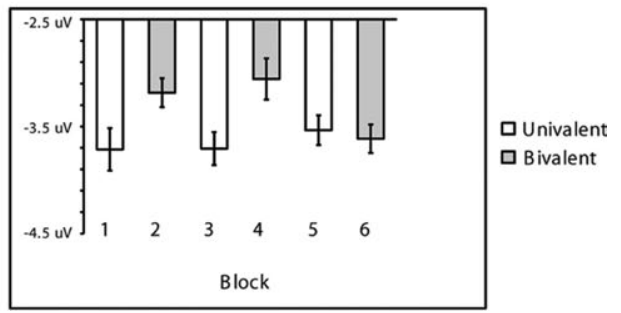

Figure 3.

Representation of whole brain analysis and electrode clusters capturing the bivalency effect. (A) Partial least squares (PLS) analyses produced Latent variable I (LVI) which captures the contrast between the first bivalent block compared to the other blocks. LVI accounts for $35 \%$ of the cross-block covariance explained by the experimental conditions $(P=0.006)$. (B) Fron-

Statistical analysis of this posterior cluster confirmed a significantly larger positive deflection to uni-uni trials than to uni-biv trials at $100-120 \mathrm{~ms}$ after stimulus onset, $t(24)=$ 2.30, $P=0.03, \eta^{2}=0.181$. This effect was also significant at the corresponding right posterior cluster (P6, P8, and PO8), $t(24)=2.34, P=0.028, \eta^{2}=0.186$.

For the 375-450-ms time window, two principle components were needed to account for $98.4 \%$ of the variance $(96 \%+2.4 \%$ separately) in the ERP difference scores. The first dipole (accounting for $96 \%$ of the variance) was located at $x, y, z=4.2,20.8,33.1$, ori: $-0.3,0.8,-0.6$. This corresponds to the dorsal anterior cingulate cortex (dACC), consistent with the fMRI BOLD responses obtained from the Woodward et al. (2008) bivalency effect study. The second dipole was located at $x, y, z=11.1$, $-51.3,15.8$, ori: $0.7,0.7,0.2$, near the posterior cingulate tal and central waveforms depicting electrophysiological responses during the bivalency effect (uni-biv trials in block 2 compared to the average uni-uni trials in blocks I and 3). (C) Block by block comparison illustrating the bivalency effect over time.

cortex. This model is the best fit for the data, and corresponds to a residual variance (RV) of $5.2 \%$ at the peak activity of these dipoles (418 ms; see Fig. 6B).

For the 500-550-ms time window, two principle components were needed to account for $98.7 \%$ of the variance $(86.4 \%+12.3 \%$ separately) in the ERP difference scores. The first dipole (accounting for $86.4 \%$ of the variance) was located at $x, y, z=-13,25.2,36.8$, ori: -0.2 , $0.9,-0.5$. This corresponds to the dACC, consistent with the fMRI BOLD responses obtained from the Woodward et al. (2008) bivalency effect study. The second dipole was located at $x, y, z=5.2,-30.9,-3.9$, ori: $0.3,0.4,0.9$, in the midbrain area. This model is the best fit for the data, and corresponds to a residual variance (RV) of $7.7 \%$ at the peak activity of these dipoles (523 ms; see Fig. 6C). 


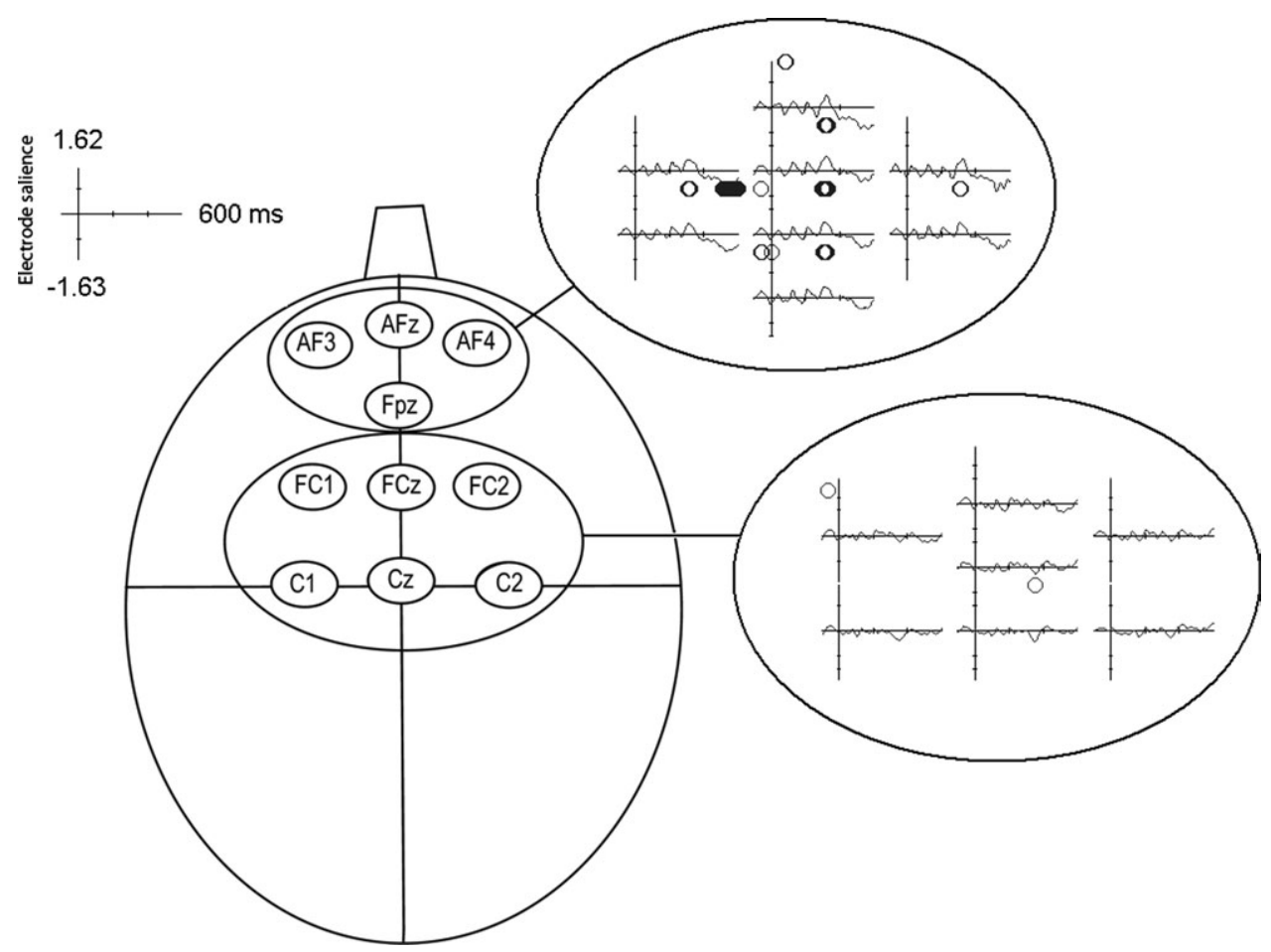

Figure 4.

Illustrates the location of electrodes chosen for the central and frontal clusters according to the standard 10/20 system. The frontal cluster consisted of areas Fpz, AFz, AF4, and AF3, while the central cluster consisted of areas $\mathrm{Cz}, \mathrm{Cl}, \mathrm{C2}, \mathrm{FCz}, \mathrm{FCl}$, and $\mathrm{FC} 2$. The electrode salience maps for the partial least squares analysis (see results section) for these clusters are also illustrated.

\section{Electrophysiology: Extended Practice and the Bivalency Effect}

To examine extended practice on the bivalency effect we return to our PLS analysis (Fig. 3A). LV1 shows a pattern across the six blocks that suggests a reduction in the size of the bivalency effect with practice, consistent with the behavioral results. To further examine this possibility, ERP amplitude difference scores at the frontal cluster were calculated to represent the difference between responses to uni-uni stimuli and uni-biv stimuli (bivalency effect). Block 1 was subtracted from block 2 (blocks 2-1), block 3 was subtracted from block 4 (blocks 4-3), and block 5 was subtracted from block 6 (blocks 6-5). If the bivalency effect is reduced with practice, then it should be largest for the first two blocks and smallest for the last two blocks, leading to a significant linear contrast over time. The difference scores (blocks 2-1, 4-3, and 6-5) were analyzed by means of repeated measures ANOVAs. Within the time windows 100-120 ms, and 500-550 ms, these ANOVAs revealed significant within-subject linear contrasts at the frontal cluster, $F(1,24)=6.955, P=0.014, \eta^{2}=0.225$, and $F(1,24)=$ 6.403, $P=0.018 ., \eta^{2}=0.211$, respectively. At $375-450 \mathrm{~ms}$, the linear contrast for this comparison was nonsignificant, $F(1,24)=2.063, P=0.164, \eta^{2}=0.079$, but in the predicted direction (i.e., the difference scores became smaller over time). Figure 5 illustrates the voltage maps that capture the bivalency effect at each time window and stage of the experiment. The voltage maps provide an illustration of the amplitude differences between univalent trials within univalent and bivalent blocks. Across time windows, the voltage maps show that the activity seen at the beginning of the experiment (block 2-1) disappears by the end of the experiment (block 6-5), as would be expected from a practice effect. These maps in concert with the aforementioned results further support that the electrophysiological responses to the bivalency effect dissipate over the course of the experiment (see Figs. 3 and 5).

\section{DISCUSSION}

When alternating between multiple tasks, if we occasionally encounter stimuli that cue two ongoing tasks (i.e., bivalent stimuli), response slowing is observed on all trials (bivalency effect). The occasional presence of bivalent 


\section{Voltage Maps}

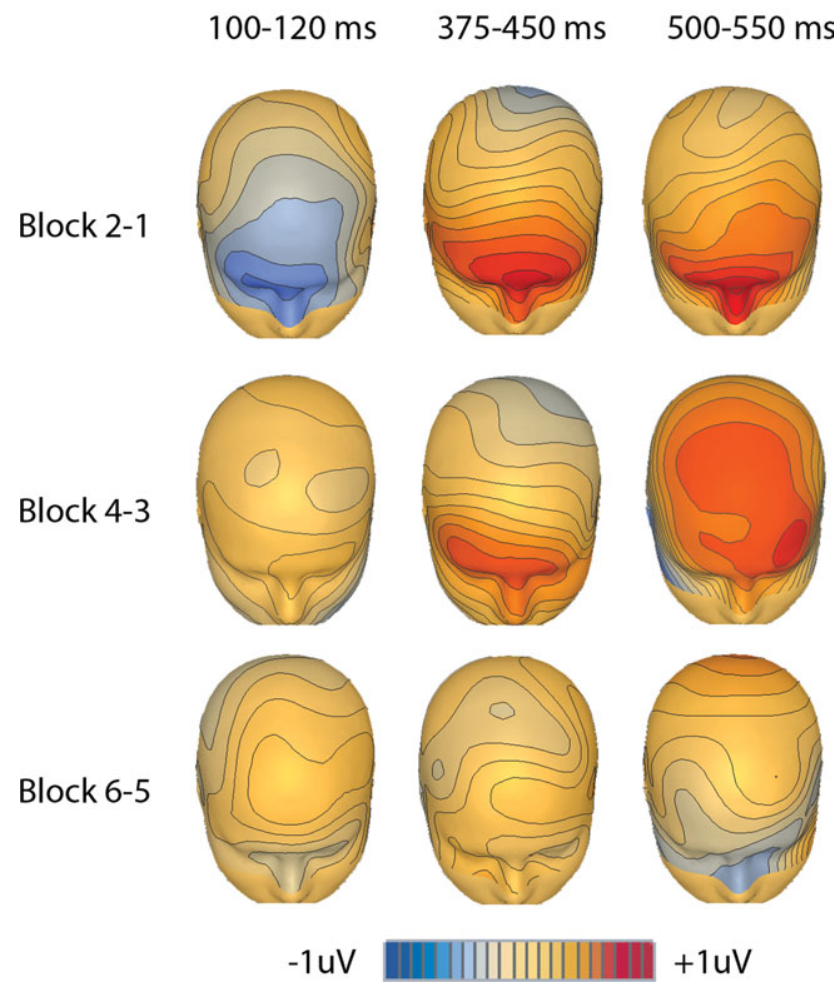

Figure 5.

Voltage topographies showing a change in electrophysiological responses for univalent trials across the experiment. Topographies are displayed for each time window of interest: 100-120 $\mathrm{ms}, 375-450 \mathrm{~ms}$, and $500-550 \mathrm{~ms}$. The bivalency effect was captured in three block sets, in which block I amplitudes were subtracted from block 2, block 3 amplitudes were subtracted from block 4, and block 5 amplitudes were subtracted from block 6 . These block sets represent changes in the bivalency effect at three stages across the experiment.

stimuli within a block may be enough to elicit an alternative blockwise response strategy (Woodward et al., 2003, 2008). There is a crucial role for the ACC in the bivalency effect as evidenced by fMRI studies (Woodward et al., 2008) although the role of the ACC has been unclear. Significantly greater ACC activation was observed on univalent trials when they occurred in a block with occasional bivalent trials, and the authors concluded that the ACC may be responsible for breaking task inertia to implement an alternative response strategy. This hypothesis is supported by other neuroimaging studies, mathematical modeling, and animal studies which have suggested that changing response strategies may be signaled by the ACC (Behrens et al., 2007; Gehring and Taylor, 2004; Hayden and Platt, 2006; Luks et al., 2002; Paus, 2001; Ridderinkhof et al., 2004; Rushworth et al., 2004; Volz et al., 2003; Williams et al., 2004).
Using a paradigm similar to that of Woodward et al. (2008), we measured ERPs to extend the fMRI findings and suggest a time course for ACC activity as it relates to the bivalency effect. This allowed us to identify components that captured the differential processing of uni-biv and uni-uni stimuli, which aids in our understanding of how we implement and maintain a new response style after encountering bivalent stimuli. In addition, we looked at how brain and behavioral responses associated with the bivalency effect changed over extended practice. Past studies have examined the bivalency effect using two univalent blocks and one bivalent block; the present paradigm examined the effects of practice by doubling the number of blocks (three univalent blocks alternating with three bivalent blocks) and increasing the number of trials per block (an additional 48 trials or 16 triplets). We present two main findings: (1) The contrast between univalent trials in bivalent compared to univalent blocks (the bivalency effect) is reflected by amplitude differences at frontal electrodes among 100-120 ms, 375-450 ms, and 500-550 ms (Fig. 3B), with sources in the ACC for the two later components (Fig. 6). (2) The bivalency effect dissipates with practice, both behaviorally and electrophysiologically (Figs. 2 and 3C).

We replicated previous behavioral results (Woodward et al., 2003, 2008), showing slower responses to univalent trials when they occurred in blocks in which occasional bivalent stimuli were presented. Furthermore, a practice effect was observed across all three tasks (color judgment, parity judgment, and case judgment) showing a decrease in the magnitude of the bivalency effect across the experimental session. These behavioral data fit nicely with the ERP results showing that the bivalency effect dissipates with extended practice.

Interestingly, the behavioral bivalency effect appeared most pronounced for color judgment trials and did not dissipate with practice to the same extent as did the case and parity judgments (see Fig. 2). A possible explanation for this effect can be attributed to the predictable trial sequence. Past work has shown that, in a predictable trial sequence, trials immediately following bivalent stimuli have longer RTs than subsequent stimuli (Meier et al., 2009). Because bivalent stimuli were always followed by color trials, it is reasonable to predict that these trials would have the longest average RTs. Thus, when comparing all color trials within bivalent blocks to all color trials within univalent blocks, we should not be surprised that we observed a more pronounced bivalency effect than for case or parity judgments across the experiment. Another possible explanation for color showing a more robust bivalency effect is that the color judgments share a feature (color) with the bivalent stimuli, a feature that is irrelevant and must be ignored or suppressed on bivalent trials. Consistent with this idea, Rey-Mermet and Meier (in press) conducted a bivalency effect study in which the color task 
A. $100-120 \mathrm{~ms}$
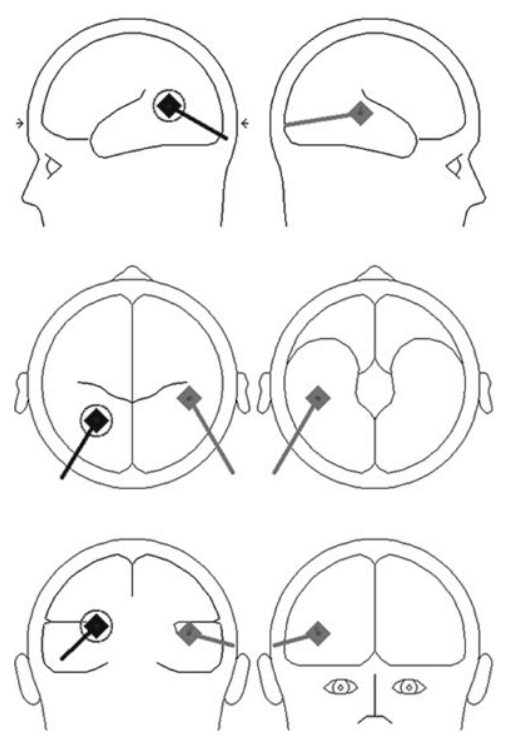

B. $375-450 \mathrm{~ms}$
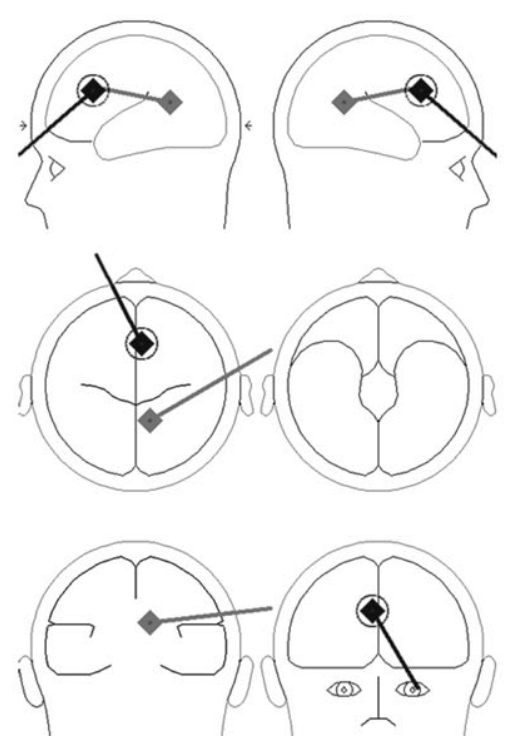

C. $500-550 \mathrm{~ms}$
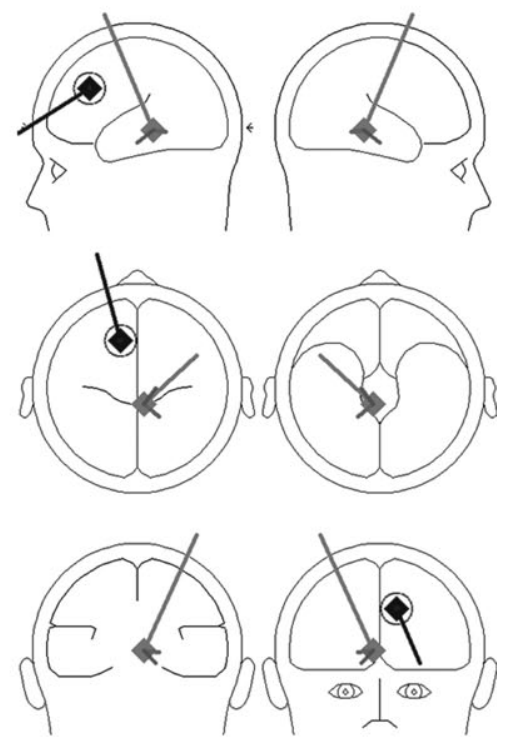

Figure 6.

Source dipole models used to explain the maximum amount of variance for the bivalency effect in each time window of interest: (A) $100-120 \mathrm{~ms}$, (B) $375-450 \mathrm{~ms}$, and (C) 500-550 ms. For each component, the first dipole accounting for most of the variance is shown in black, and the second dipole is shown in grey. The first dipole for the 100-120 ms window explains $95.4 \%$ of

occurred before the case task and after the parity task; the color task appeared to produce the largest bivalency effect regardless of sequence position ${ }^{1}$. It may be that the whole task set for the color trials is suppressed in response to the bivalent stimuli, and that this suppression has a longer lasting effect than the reconfiguration signaled by the ACC. The idea of task set suppression is supported by an ERP study comparing univalent stimuli to congruent and incongruent bivalent stimuli (Hsieh and Liu, 2008). ERPs for congruent and incongruent bivalent stimuli did not differ from one another although both differed from the univalent stimulus. The authors proposed that the irrelevant feature on a bivalent trial leads to the suppression of the task set rather than suppression at the response level. In the case of our experiment, color was always the irrelevant feature on the bivalent trials, possibly leading to a more robust bivalency effect for the color task.

The main purpose of this article is to expand on the existing behavioral and fMRI work by providing high tem-

${ }^{1}$ When the sequence of tasks proceeded from color to parity to case the bivalency effect was 100, 48, and $38 \mathrm{~ms}$, respectively. When the sequence proceeded from parity to color to case the bivalency effect was 62,83 , and $51 \mathrm{~ms}$, respectively. While the color task appeared to produce the largest bivalency effect in both sequences, pairwise tests were not performed to confirm this observation. the variance and is located in the temporal-parietal area; the first dipole for the $375-450 \mathrm{~ms}$ window explains $96 \%$ of the variance and is located in the ACC, and the first dipole for the $500-550 \mathrm{~ms}$ window explains $86.4 \%$ of the variance and is located in the ACC.

poral resolution time-course analyses. The ERP measurements captured the bivalency effect at the frontal cluster and revealed three relevant time windows: 100-120 ms, $375-450 \mathrm{~ms}$, and $500-550 \mathrm{~ms}$. At $100-120 \mathrm{~ms}$, the electrophysiological response was sensitive to the bivalency manipulation in the first set of univalent versus bivalent blocks (blocks 2-1), and this response was not apparent at the later block comparisons. Source analysis revealed that this component may be a reflection of activity from an area near the left temporal-parietal junction (TPJ). At the 375-450 ms- and 500-550-ms time windows, the bivalency effect was observed for the first two block-set comparisons (blocks 2-1 and blocks 4-3), but not for the last comparison (blocks 6-5). Source analysis revealed that dACC activity is likely responsible for modulating the bivalency effect behavioral changes during these time windows.

It is interesting that the bivalency effect contrast at the early component appears to dissipate earlier with practice than do the later components. The 100-120-ms time window may reflect additional early visual-perceptual processing needed during bivalent blocks. Source modeling identified a possible source for this time window near the area of the TPJ, which has been known to be involved in early visual extraction of object components. For instance, in a global/local task involving the identification of the smaller (local) letters that make up a larger (global) letter, the left TPJ plays a role in early visual processing (Evans et al., 2000). When bivalent stimuli are first detected 
within the first bivalent block of our experiment, they are unexpected and it may not be clear that bivalent features will be present only on case-judgment trials. In the first bivalent block, it may be necessary to analyze stimuli at a more feature (local) level to determine which features are relevant to the task. If this is the case, then this analysis of stimuli at a feature level may extend to all trials within bivalent blocks, including parity decisions, which do not share any features with bivalent stimuli. For example, given the surprising colored letter on the case task, the system cannot rule out the possibility of an equivalent surprise on the parity task. This uncertainty triggers a more in-depth analysis at the feature level. This change in visual processing may disrupt the fluency of early visual processing, and require additional TPJ activity to help extract critical visual information. In later bivalent blocks, when participants have clearer expectations about where to expect bivalent stimuli (i.e., on occasional case-judgment trials), it is possible that the TPJ receives feedback from higher order centers (such as the dACC), and additional visual extraction is no longer necessary. The latency of this early component (100-120 ms) is consistent with the posterior P1, an early visual component associated with extrastriate cortex. However, our source modeling does not put the source in that area and moreover, the P1 has not been shown to be sensitive to task-switching manipulations (Wylie et al., 2003). Given that this is a blockwise effect, such an early response on individual trials is not entirely surprising because previous bivalent stimuli set the system into a more cautious state, which could affect very early processes on subsequent trials.

Studies examining switch versus repeat trials and studies comparing univalent and bivalent stimuli share some similarities with our later components (375-450 ms and 500-550 ms). For example, the comparison between univalent and bivalent trials noted by Poulsen et al. (2005) is similar to the differences we found between uni-uni and uni-biv stimuli. Those authors found a more positive deflection for bivalent relative to univalent trials between 300 and $600 \mathrm{~ms}$, and our ERP results show that uni-biv stimuli are more positive relative to uni-uni stimuli during these time frames. Remember that in the Poulsen et al. (2005) study, the ERP waveforms contrasted responses to univalent versus bivalent stimuli. Our ERP waveforms did not include the bivalent trials; rather, our contrast was between univalent trials in univalent blocks versus bivalent blocks. Thus, the similarities in the ERP components may suggest an overlap in processes related to the bivalency effect and conflict generated by the bivalent stimuli. In the bivalency effect, this conflict may carry over to the univalent trials. Detection of conflict may signal the dACC to implement an alternative response strategy, and this response strategy may include suppression of irrelevant features as well as more careful perceptual processing on the other tasks. After sufficient practice with bivalent stimuli it becomes easier to extract the relevant and ignore the irrelevant information, and this more efficient processing leads to the dissipation of processing differences across the univalent trials, consistent with our behavioral and ERP results. By that time, the ACC signals are no longer necessary to trigger additional control. The broad time windows of these later components make them good candidates to reflect the activity of the generators that produce the BOLD response in AACC that is associated with the bivalency effect (Woodward et al., 2008). This hypothesis is further supported by the source modeling that we performed suggesting dACC sources for these components.

There are also similarities between our later components at $375-450 \mathrm{~ms}$ and $500-550 \mathrm{~ms}$ and ERP components revealed in task-switching experiments that compare task repeat versus switch trials; those components are also located at frontal electrodes within a similar time window of 300-700 ms (Lorist et al., 2000; Hsieh and Liu, 2009; Wylie et al., 2003; Hsieh and Chen, 2006). It is possible that the similarities across these task-switching experiments, the bivalent versus univalent contrast (Poulsen et al., 2005), and our bivalency effect results indicate that the frontal components are all accessing processes related to the task-switching demands inherent in all three types of experiments.

Interestingly, we did not observe an ERP distinction between uni-biv and uni-uni stimuli within the time window of the N2 (200-350 ms). The N2 has been identified in a number of ERP studies as dissociating between conflict and nonconflict trials (Yang and Wang, 2002; Kong et al. 2000; Wang et al., 2002) and has a hypothesized generator localized in the ACC (van Veen and Carter, 2002). Studies comparing univalent to bivalent stimuli also do not find ERP differences at the N2 (Poulsen et al., 2005; Hsieh and Liu, 2008) suggesting that bivalency and the bivalency effect present a special case of conflict that influences later task-switching processes.

The dorsal ACC (Brodmann's area 32) fits well with our data and was the primary area of interest in the fMRI work on the bivalency effect. Woodward et al. (2008) also noted peak activation associated with the bivalency effect at Brodmann's area 9, which is part of the prefrontal cortex (PFC). The PFC is often associated with higher-order mental processes including top-down attentional control (Liston et al., 2006) and task preparation (Sohn et al., 2000; MacDonald et al., 2000; Yeung et al., 2006). While our source modeling analysis places the source of our frontal effects in the dACC, we cannot rule out that the PFC may be contributing to our frontal effects.

Another consideration when interpreting the current results is the relation between the initial univalent blocks and the effect of bivalent stimuli. Participants performed three univalent blocks before encountering bivalent stimuli in the fourth block (following two practice univalent blocks and one experimental univalent block). If participants had encountered bivalent stimuli in the first experimental block, immediately after the two univalent practice blocks, the bivalency effect may have dissipated more quickly over the course of the remaining experimental 
blocks. This counterbalancing was not done in the present experiment and could be addressed in future work interested in the relation between initial practice and the bivalency effect.

\section{CONCLUSIONS}

Using an unbiased whole-brain statistical approach (PLS; Lobaugh et al., 2001; McIntosh et al., 1996) coupled with conventional componential analyses, we provide the first ERP account for the bivalency effect and identify three significant components at a frontal electrode cluster within time windows 100-120 ms, 375-450 ms, and 500$550 \mathrm{~ms}$. We believe the bivalency effect is captured by two processes: additional extraction of visual features on all trials that is triggered by irrelevant cues in bivalent stimuli (100-120 ms) and suppression of processing carried over from irrelevant cues (375-450 ms and 500-550 ms). We showed a correspondence between behavioral and ERP responses that provide additional temporal information related to the dissipation of the bivalency effect with practice. Our results extend the fMRI findings on the bivalency effect (Woodward et al., 2008) in that the activity reflected by the frontal electrode cluster is consistent with dACC activity contrasting univalent trials in bivalent blocks to univalent trials in univalent blocks within time windows 375$450 \mathrm{~ms}$ and 500-550 ms.

\section{REFERENCES}

Behrens TE, Woolrich MW, Walton ME, Rushworth MF (2007): Learning the value of information in an uncertain world. Nat Neurosci 10:1214-1221.

Düzel E, Habib R, Schott B, Schoenfeld A, Lobaugh N, McIntosh AR, Scholz M, Heinze HJ (2003): A multivariate, spatiotemporal analysis of electromagnetic time-frequency data of recognition memory. NeuroImage 18:185-197.

Evans MA, Shedden JM, Hevenor SJ, Hahn MC (2000): The effect of variability of unattended global and local information: Evidence for lateralization at early stages of processing. Neuropsychologia 38:225-239.

Gehring WJ, Taylor SF (2004): When the going gets tough, the cingulate gets going. Nat Neurosci 7:1285-1287.

Hayden BY, Platt ML (2006): Fool me once, shame on me - fool me twice, blame the ACC. Nat Neurosci 9:857-859.

Hsieh S, Chen P (2006): Task reconfiguration and carryover in task switching: An event-related potential study. Brain Res 1084:132-145.

Hsieh S, Liu, H (2008): Electrophysiological correlates of task conflicts in task-switching. Brain Res 1203:116-125.

Hsieh S, Liu, H (2009): Electrophysiological evidence of the adaptive task-set inhibition in task switching. Brain Res 1255:122131.

Karayanidis F, Coltheart M, Michie PT, Murphy, K (2003): Electrophysiological correlates of anticipatory, and poststimulus components of task switching. Psychophysiol 40:329-348.
Kerns JG, Cohen JD, MacDonald AW III, Cho RY, Stenger VA, Carter CS (2004): Anterior cingulate conflict monitoring and adjustments in control. Science 303:1023-1026.

Kong J, Wang Y, Zhang W, Wang H, Wei H, Shang H, Yang X, Zhang D (2000) Event-related brain potentials elicited by a number discrimination task. Neuroreport 11:1195-1197.

Liston C, Miller MM, Goldwater DS, Radley JJ, Rocher AB, Hof PR (2006): Stress-induced alterations in prefrontal cortical dendritic morphology predict selective impairments in perceptual attentional set-shifting. J Neurosci 26:7870-7874.

Liu X, Banich MT, Jacobson BL, Tanabe JL (2004): Common and distinct neural substrates of attentional control in an integrated Simon and spatial Stroop task as assessed by event-related fMRI. NeuroImage 22:1097-1106.

Lobaugh NJ, West R, Mcintosh AR (2001): Spatiotemporal analysis of experimental differences in event-related potential data with partial least squares. Psychophysiology 38:517-530.

Lorist M, Klein M, Nieuwenhuis S, De Jong R, Mulder G, Meijman, T (2000): Mental fatigue and task control: Planning and preparation. Psychophysiology 37:614-625.

Luks TL, Simpson GV, Feiwell RJ, Miller WL (2002): Evidence for anterior cingulate cortex involvement in monitoring preparatory attentional set. NeuroImage 17:792-802.

MacDonald AW, Cohen JD, Stenger VA, Carter C (2000): Dissociating the role of the dorsolateral prefrontal and anterior cingulate cortex in cognitive control. Science 288:18351838.

McIntosh AR, Bookstein FL, Haxby JV, Grady CL (1996): Spatial pattern analysis of functional brain images using Partial Least Squares. Neuroimage 3:143-157.

Meier B, Woodward TS, Ray-Mermet A, Graf P (2009): The bivalency effect in task-switching: General and enduring. Can J Exp Psychol 63:201-210.

Metzak PD, Meier B, Graf P, Woodward TS, (submitted): Not just a surprise: The bivalency effect in task switching represents the adaptive tuning of a response style.

Milham MP, Banich MT, Webb A, Barad V, Cohen NJ, Wszalek T, Kramer AF (2001): The relative involvement of anterior cingulate and prefrontal cortex in attentional control depends on nature of conflict. Cogn Brain Res 12:467-473.

Paus T (2001): Primate anterior cingulate cortex: Where motor control, drive and cognition interface. Nature Reviews. Neuroscience 2:417-424.

Poulsen C, Luu P, Davey C, Tucker DM (2005): Dynamics of task sets: Evidence from dense-array event-related potentials. Cogn Brain Res 24:133-154.

Rey-Mermet A, Meier B (in press): The bivalency effect: Adjustment of cognitive control without response set priming. Psychol Res. DOI: 10.1007/s00426-011-0322-y.

Ridderinkhof KR, Ullsperger M, Crone EA, Nieuwenhuis S (2004): The role of the medial frontal cortex in cognitive control. Science 306:443-447.

Rushworth MF, Walton ME, Kennerley SW, Bannerman DM (2004): Action sets and decisions in the medial frontal cortex. Trends Cogn Sci 8:410-417.

Stemmer B, Segalowitz SJ, Witzke W, Schönle PW (2003): Error detection in patients with lesions to the medial prefrontal cortex: An ERP study. Neuropsychologia 42:118-130.

Slagter HA, Weissman DH, Giesbrecht B, Kenemans JL, Mangun GR, Kok A, Woldorff MG (2006): Brain regions activated by endogenous preparatory set shifting as revealed by fMRI. Cogn Affect Behav Neurosci 6:175-189. 
Sohn M, Anderson JR (2000): Task preparation and task repetition Two-component model of task switching. J Exp Psychol 130:764-778.

Ullsperger M, von Cramon DY (2001): Subprocesses of performance monitoring: A dissociation of error processing and response competition revealed by event-related fMRI and ERPs. Neuroimage 14:1387-1401.

van Veen V, Carter CS (2002): The timing of action-monitoring processes in the anterior cingulate cortex. J Cogn Neurosci 14:593-602.

van Veen V, Cohen JD, Botvinick MM, Stenger VA, Carter CS (2001): Anterior cingulate cortex, conflict monitoring, and levels of processing. NeuroImage 14:1302-1308.

Volz KG, Schubotz RI, Von Cramon Y (2003): Predicting events of varying probability: Uncertainty investigated by fMRI. NeuroImage 19:271-280.

Wang Y, Wang H, Cui L, Tian S, Zhang Y (2002): The N270 component of the event-related potential reflects supramodal conflict processing. Neurosci Lett 332:25-28.

Williams ZM, Bush G, Rauch SL, Cosgrove GR, Eskandar EN (2004): Human anterior cingulate neurons and the integration of monetary reward with motor responses. Nat Neurosci 7:1370-1375.
Woldorff MG (1993): Distortion of ERP averages due to overlap from temporally adjacent ERPs: Analysis and correction. Psychophysiology 30:98-119.

Woodward TS, Meier B, Tipper C, Graf P (2003): Bivalency is costly: Bivalent stimuli elicit cautious responding. J Exp Psychol 50:233-238.

Woodward TS, Metzak PD, Meier B, Holroyd CB (2008): Anterior cingulate cortex signals the requirement to break inertia when switching tasks: A study of the bivalency effect. NeuroImage 40:1311-1318.

Woodward TS, Ruff CC, Ngan ET (2006): Short- and long-term changes in anterior cingulate activation during resolution of task-set competition. Brain Res 1068:161-169.

Wylie GR, Javitt DC, Foxe JJ (2003): Task switching: A high-density electrical mapping study. NeuroImage 20:2322-2342.

Wylie GR, Murray MM, Javitt DC, Foxe JJ (2009): Distinct neurophysiological mechanisms mediate mixing costs and switch costs. J Cogn Neurosci 21:105-118.

Yang J, Wang Y (2002): Event-related potentials elicited by stimulus spatial discrepancy in humans. Neurosci Lett 326:73-76.

Yeung N, Nystrom LE, Aronson JA, Cohen JD (2006): Betweentask competition and cognitive control in task switching. J Neurosci 26:1429-1438. 